\title{
High level EGFR amplification in a newly established glioblastoma cell line 170-MG-BA
}

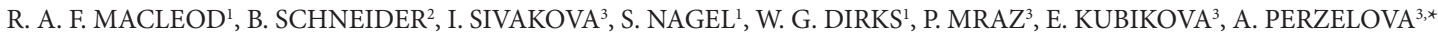

${ }^{1}$ Department of Cell Cultures, DSMZ-German Collection of Microorganisms and Cell Cultures, Braunschweig, Germany; ${ }^{2}$ Institute of Pathology and Molecular Pathology, University Medicine Rostock, Rostock, Germany; ${ }^{3}$ Department of Anatomy, Faculty of Medicine, Comenius University, Bratislava, Slovakia

${ }^{*}$ Correspondence: anna.perzelova@fmed.uniba.sk

Received April 27, 2018 / Accepted July 11, 20187

\begin{abstract}
Glioblastoma multiforme is a highly invasive and incurable primary brain tumor. The most frequent genetic alteration therein is amplification of the epidermal growth factor receptor (EGFR) gene, the target of current clinical trials. However, EGFR amplification is poorly represented in glioblastoma cell lines. From the 30 cultures attempted herein, we were able to establish two glioblastoma permanent cell lines. The remaining cultures showed limited life span and underwent senescence between passage numbers (PN) 8 to 15. Our newly established glioblastoma cell lines, designated 170-MG-BA and 538-MGBA, both originated between PN 3 and 5 when areas of smaller, more rapidly proliferating cells appeared. Both cell lines showed similar rates of growth, moderate morphological differences, cytoskeletal heterogeneity and multiple chromosome rearrangements. Analysis by molecular cytogenetics and comparative genomic hybridization (aCGH) revealed two copies of a stable marker chromosome in 170-MG-BA cells effecting focal amplification at 7q11 of the EGFR locus. Comparative RqPCR analysis confirmed that EGFR was uniquely highly expressed in 170-MG-BA cells. Combined targeted expression analysis and aCGH data excluded the recurrent EGFRvIII activating mutation. In contrast, EGFR expression in 538-MG-BA cells which lacked genomic EGFR amplification was not raised. Immunofluorescent staining showed high EGFR protein expression only in the 170-MG-BA cells. Cytogenetic, genomic and transcriptional analyses then confirmed high-level genomic amplification and transcriptional upregulation of wild type EGFR in 170-MG-BA; the first conventional cell line model for investigating the biology and targeted therapy of this key alteration in glioblastoma. Both cell lines are freely available from the DSMZ cell repository.
\end{abstract}

Key words: glioblastoma cell lines, intermediate filaments, EGFR, gene amplification, EGFR-targeted therapy

Gliomas are primary brain tumors with varying degrees of malignancy. Glioblastoma multiforme is one of the most malignant gliomas and confers a lethal prognosis. Treatment resistance has been attributed to genetic heterogeneity and numerous studies currently address this phenomenon, notably by investigating cancer stem cells and identifying cancer initiating cells. High level genomic EGFR amplification $\left(\mathrm{EGFR}^{\mathrm{HIGH}}\right)$ is the most frequent genomic alteration characterizing glioblastoma and affects about $40 \%$ cases, with a causal role in pathogenesis and treatment resistance [1]. EGFR is currently undergoing evaluation as a clinical target where trials have thrown up new challenges $[2,3]$. Unfortunately, $\mathrm{EGFR}^{\mathrm{HIGH}}$ is seemingly unstable in conventional cell lines and can be only maintained short-term, such as the approximately five passages recently reported [4]. Although the DK-MG cell line bears high level (mutant) EGFR ampli- fication, this is heterogeneous and affects a subpopulation of cells, with others lacking amplification $[5,6]$. Hitherto, one glioma cell line has been described with homogeneous EGFR amplification, SKMG-3, but it has only moderate amplification levels [7].

Dearth of EGFR ${ }^{\mathrm{HIGH}}$ cell lines has necessitated undue reliance on ectopic expression models $[8,9]$. While modified culture protocols have been developed to preserve wild type EGFR $^{\text {HIGH }}$ stability, notably withdrawal of receptor ligand EGF [9], no conventional glioblastoma cell lines exist which preserve this key alteration to serve as a convenient laboratory tool for developing targeted therapies [9]. While more recent neurosphere models preserve additional glioma features, conventional cell lines are better suited for use in standardized drug response assays because these facilitate quantitative comparisons. $\mathrm{EGFR}^{\mathrm{HIGH}}$ is frequently $(40-50 \%)$ 
accompanied by expression of different variants/mutants, most often EGFRvIII [1, 10] - a deletion variant lacking exons 2-to-7 encoding the extracellular domain, an alteration which prevents EGF binding. EGFRvIII thus confers ligand-independent signaling by allowing heterodimerization to wild-type EGFR, thereby activating pathways other than those controlled by wild-type EGFR $[11,12]$.

Of three barriers which have impeded the successful application of cancer cell lines - namely, mycoplasma infection, cross-contamination and unavailability, the former two have been addressed by technical advances first implemented by public cell repositories. The last remains a chronic problem, however, leaving several key entities under/un-represented by in vitro models [13]. Reasons for this dearth are the respective reluctance of some originators to make rare in vitro models freely available and of certain tumors to undergo establishment in vitro. Moreover, while several widely used glioma cell lines are available, retrospective authentication has revealed that too many of these bear questionable or false identities $[14,15,16]$. Thus, the unavailability of reliable glioma models covering the disease spectrum illustrates a chronic and widespread problem [17].

The foregoing considerations mandate establishment of new glioblastoma cell lines with reliable provenances, in the current context notably EGFR ${ }^{\mathrm{HIGH}}$ glioblastoma models. In this study we describe the establishment of two new adherent glioblastoma cell lines; one with high level EGFR amplification and expression to serve as candidate to fill this hitherto unrequited need.

\section{Materials and methods}

Glioma samples and cell cultures. Tumor brain material was kindly provided by the Department of Neurosurgery, Derer's Hospital, Bratislava. Experiments with human brain biopsies were performed according to Slovak laws 272/1994, 76/2004 and approved by the Ethical Committee of UNB Bratislava. Accordingly, bioptic samples collected between 1993 and 2011 were cut into small pieces and seeded in uncoated plastic dishes $\left(25 \mathrm{~cm}^{2}\right)$. Culture medium consisted of MEM with glutamine, non-essential amino acids (Biocom, Brno, Czech Republic) and 10\% fetal calf serum (Sigma, Darmstadt, Germany). Primary cultures and/or the cells in early passages were cryopreserved in liquid nitrogen. For cell line establishment, we recultured 30 cryopreserved glioblastoma cultures (from donor patients aged 39 to 74 years). From these cultures two permanent cell lines were established; both from bioptic samples of primary glioblastoma multiforme grade IV. Cell lines were sub-cultivated up to (absolute) passage number (PN) 50 to confirm immortalization.

The remaining cell lines used in this study were drawn from the DSMZ reference cell repository. Authentication was performed by DNA STR profiling according to standard DSMZ protocols (www.dsmz.de). STR profiles are available from the authors on request.

Immunofluorescence staining. Cells grown on coverslips were rinsed with PBS, fixed in methanol-acetone $(1: 1)$ solution for $15 \mathrm{~min}$ at $-15^{\circ} \mathrm{C}$ and used for indirect and double immunofluorescence staining. For IF proteins examination, we used the following antibodies: against GFAP, clone GF-01, 1:100, (Exbio, Prague, Czech Republic), clone G-A-5-Cy3 conjugate, 1:400, (Sigma, Saint Louis, USA) and polyclonal sera to GFAP, 1:100, (Dako, Glostrup, Denmark); to vimentin clone V9, 1:100 (Sigma); to cytokeratins monoclonal anti-pan CK types: $1,4,5,6,8,10,13,18,19$, (Sigma); to neurofilaments clone NF-01, 1:100, (Exbio); to nestin clone 25, 1:50, (BD Biosciences, New Jersey, USA) and polyclonal sera to nestin 1:100, (Millipore, Temecula, USA). EGRF staining was performed with monoclonal antibodies A-10, 1:50 (Santa Cruz, Biotechnology, Dallas, USA) on cells fixed with cold methanol for $15 \mathrm{~min}$ at $-15^{\circ} \mathrm{C}$. Secondary fluorescein- and rhodamine-conjugated antibodies were purchased from Sigma and Sevapharma (Prague, Czech Republic). Cells for indirect immunofluorescence were incubated $1 \mathrm{~h}$ with primary and $30 \mathrm{~min}$. with secondary antibodies. Double labeling for GFAP/Vim, GFAP/CK, GFAP/Nes, and CK/Nes was performed with primary, and afterwards with appropriate mixtures of secondary antibodies for $1 \mathrm{~h}$ and $30 \mathrm{~min}$, respectively; EGFR/GFAP with primary antibodies to EGFR for $1 \mathrm{~h}$, secondary fluorescein-conjugated antibodies for $30 \mathrm{~min}$ and G-A-5-Cy3 conjugate for $30 \mathrm{~min}$. Nuclei were stained with Hoechst 33258 fluorochrome $(5 \mu \mathrm{g} / \mathrm{ml}$ in PBS, Sigma) for $1 \mathrm{~min}$. To determine the percentage of immunoreactive cells, 30 fields were enumerated at $200 \times$ magnification, equally distributed over the coverslips fixed at different DIV and passage numbers. Fluorescence microscopy was performed using an Olympus BX51 microscope (Hamburg, Germany).

Growth kinetics. Proliferation rates were measured in 170-MG-BA and 538-MG-BA cell lines at PN 45-50. Accordingly, growth curves were constructed from counts of the harvested cells. For counting we used the cells from five dishes in DIV 1 to 7 . Chamber cell counting was accomplished by enumerating 20 marked microscopic fields.

Cytogenetic analysis. Cell lines were harvested and analyzed conventionally and by Spectral Karyotyping (SKY) [18]. Bacterial artificial chromosome (BAC) clone

Cell lines related to clinical data of patients with diagnosed glioblastoma multiforme.

\begin{tabular}{lcccccc}
\hline Cell line & Age & Sex & Brain localization & Date of biopsy & Cryopreserved & Cell line origin \\
\hline 170-MG-BA & 74 & F & temporal lobe & June 1995 & November 2015 \\
538-MG-BA & 49 & M & temporal lobe & April 2002 & years & 13 years \\
\hline
\end{tabular}


RP5-109e12 which carries EGFR ${ }^{4}$ was kindly supplied by Dr Sabine Riethdorf, Universitätsklinikum Hamburg-Eppendorf. The remaining BAC and fosmid clones were purchased from BACPAC Resources, Children's Hospital, Oakland, CA, USA, and DNA labeled by nick translation with d-UTP fluors (Dyomics, Jena, Germany). Fluorescence in situ hybridization (FISH) images were captured by Zeiss Axioplan microscope (Oberkochen, Germany) configured to a SKY system (ASI Ltd, Migdal Haemek, Israel) and FISH as previously described [19].

Array comparative genomic hybridization (aCGH). aCGH was performed as previously described [20]. CytoScan High Density Arrays which comprise parallel oligonucleotide and SNP probes (Affymetrix, High Wycombe, UK) were used to measure genomic copy number and losses of heterozygosity (LOH). DNA was prepared using the Qiagen Gentra Puregene Kit (Hilden, Germany). Labeling, hybridization and washing were performed with recommended kits and following manufacturers ' protocols. Quality control criteria were those defined by the manufacturer. Data was analyzed by Chromosome Analysis Suite software version 2.0.1.2 (Affymetrix).

Polymerase chain-reaction (PCR) analysis. Total RNA was extracted from cell line samples using TRIzol reagent (Invitrogen, Darmstadt, Germany). cDNA was synthesized from $1 \mu \mathrm{g}$ RNA by random priming using Superscript II (Invitrogen). Real-time quantitative (RQ)-PCR analysis was performed with the 7500 Real-time System, using commercial buffer and primer sets (Applied Biosystems, Darmstadt, Germany). We analyzed the transcript of TATA box binding protein (TBP) for normalization of expression levels, and primer sequences are available from the authors.

DNA profiling. DNA profiling used fluorescence-PCR combined with capillary electrophoresis to exclude cross contamination STR [as detailed - 21]. Briefly, the PowerPlex VR 1.2 system (Promega, Mannheim, Germany) was set to run dual-color DNA profiling, thus enabling simultaneous single-tube amplification of 8 polymorphic STR loci plus amelogenin (gender). Loci were amplified by oligonucleotides labeled with the Beckman/Coulter dye D3 (green; Sigma-Aldrich, Munich/Germany), while the STR loci D16S539, D7S820, D13S317 and D5S818 primers were labeled with D2 (black). Data was analyzed by CEQ 8000 software (Beckman-Coulter, Krefeld, Germany), permitting automatic assignment of genotypes and export of resulting numeric allele codes into the reference DNA database of the DSMZ. This enabled scrutiny against a universal comprehensive database compiled with other large cell banks.

EGFRvIII mutation expression analysis. CDNA was generated by transcribing $1 \mu \mathrm{g}$ RNA using QuantiTect Reverse Transcription Kit (Qiagen) following the manufacturer's protocol. Quantitative PCR (qPCR) was performed using SensiFAST Probe HiROX-Kit (Bioline, Luckenwalde, Germany) and self-designed primer/probe-combinations specific for EGFRvIII and TBP as endogenous control (primer
Table 1. The percentage of positive cells in glioblastoma cell lines at different passages (ps): indirect immunofluorescence staining for IF.

\begin{tabular}{lccccc}
\hline Cell lines & IF & $\mathbf{5} \mathbf{p s}$ & $\mathbf{1 0} \mathbf{p s}$ & $\mathbf{2 0} \mathbf{p s}$ & $\mathbf{5 0} \mathbf{p s}$ \\
\hline 170-MG-BA & GFAP & $50-70$ & $10-15$ & $1-2$ & 0 \\
& Vim & 100 & 100 & 100 & 100 \\
& CK & $30-40$ & $10-20$ & $1-3$ & 0 \\
& Nes & $50-70$ & $50-70$ & $50-70$ & $70-80$ \\
$538-M G-B A$ & NF & 0 & 0 & 0 & 0 \\
& GFAP & 0 & 0 & 0 & 0 \\
& Vim & 100 & 100 & 100 & 100 \\
& CK & 0 & 0 & $1-2$ & $3-5$ \\
& Nes & $70-80$ & $70-80$ & $70-80$ & $70-80$ \\
& NF & 0 & 0 & 0 & 0 \\
\hline
\end{tabular}

and probe sequences are given in Supplementary Table 1). Analysis was performed by StepOne Plus Realtime-PCR System with StepOne Software v2.1 (Applied Biosystems).

\section{Results}

Morphology of glioblastoma cell lines. Morphological features of cell lines were assessed with inverse phasecontrast microscopy performed on living cells in culture and are shown in Figures 1A-D. During early passages large flat cells predominated in 538-MG-BA cultures, with long, process-bearing astroglial cells notably absent. Areas of smaller flat and spindle shaped cells appeared between PN 3-5 (Figure 1A) and these overgrew remaining cells during subsequent passages. Cultures became more homogeneous between PN 15-20, and after PN 50 most cells became spindle shaped (Figure 1B). Cells in 170-MG-BA cultures were morphologically more heterogeneous until PN 20. In early passages, they contained a subpopulation of long process-bearing cells. The variable morphological features of astroglial cells were revealed by immunostaining with GFAP antibodies (Figures 3J-L)). Similarly, in 170-MG-BA cultures, areas of smaller cells appeared during PN 3-5 and these had mainly spindle morphology by PN 7(Figure 1C). The 170-MG-BA cell line became more homogeneous between PN 40-50 when it comprised mainly epitheloid and spindle shaped cells (Figure 1D).

Immunofluorescence. The results of indirect immunofluorescence staining for GFAP, vimentin (Vim), cytokeratins (CK), nestin (Nes) and nerofilaments (NF) are summarized in Table 1. GFAP-positive cells were detected only in the 170-MG-BA cell line in early passages. Their number decreased subsequently and completely disappeared after PN 20 (Figures 3J-L), while 538-MG-BA remained GFAPnegative. In contrast, vimentin was expressed in both cell lines. Further glial non-specific CK was present in early passages in 170-MG-BA, where the mainly flat large cells were CK-positive (Figure $3 \mathrm{G}$ ) but these cells subsequently disappeared. In 538-MG-BA cultures, a small subpopulation 

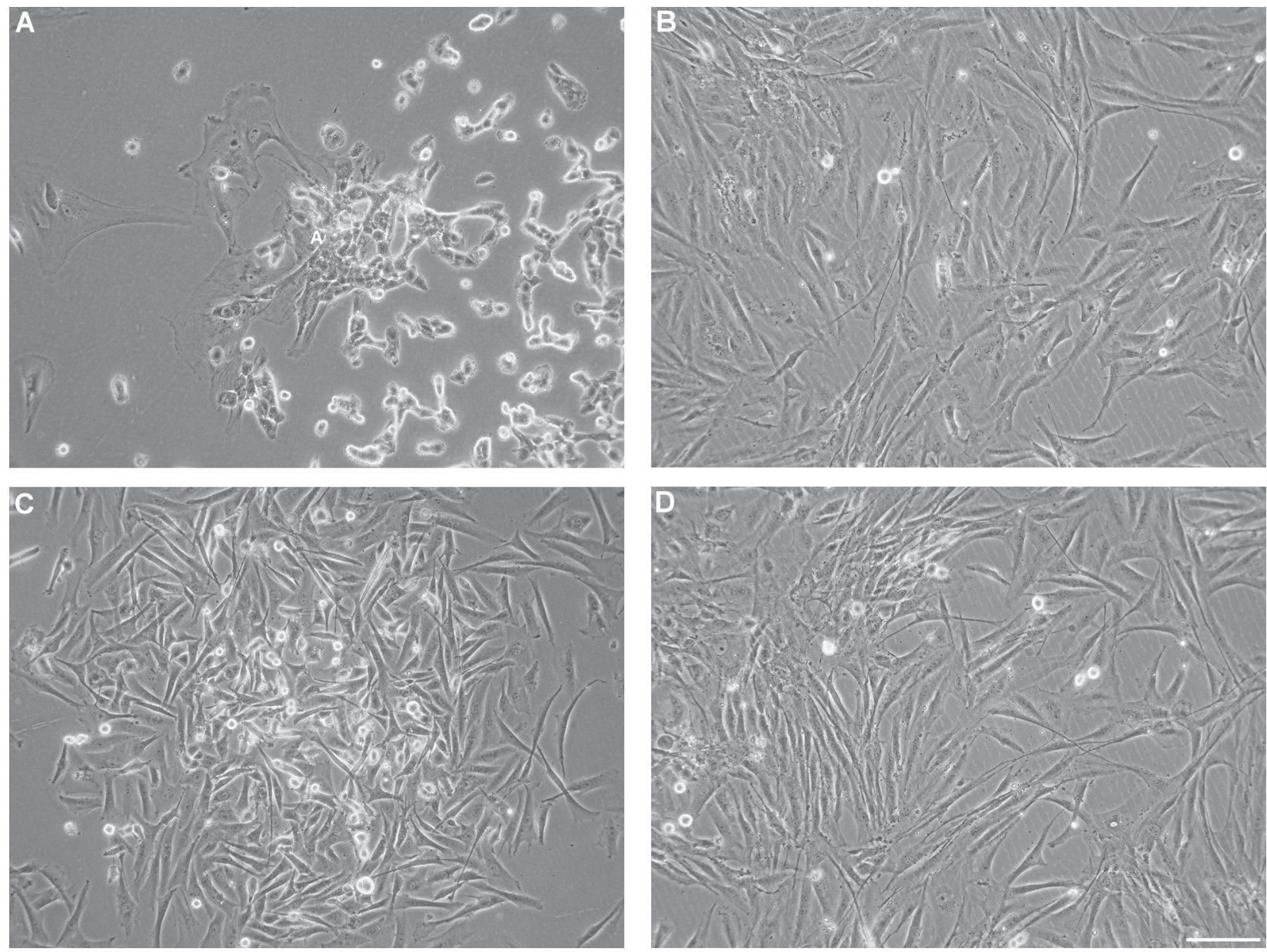

Figure 1. Morphological features of glioblastoma cell lines and living cells by phase-contrast microscopy. 538-MG-BA: Areas of smaller and several large flat cells at PN 4 (A), morphology of cell line at PN 50 (B). 170-MG-BA: Area of smaller mainly spindle-shaped cells at 7 passage (C), epitheloid and spindle-shaped cells at PN 50 (D). Scale bar: $100 \mu \mathrm{m}$.

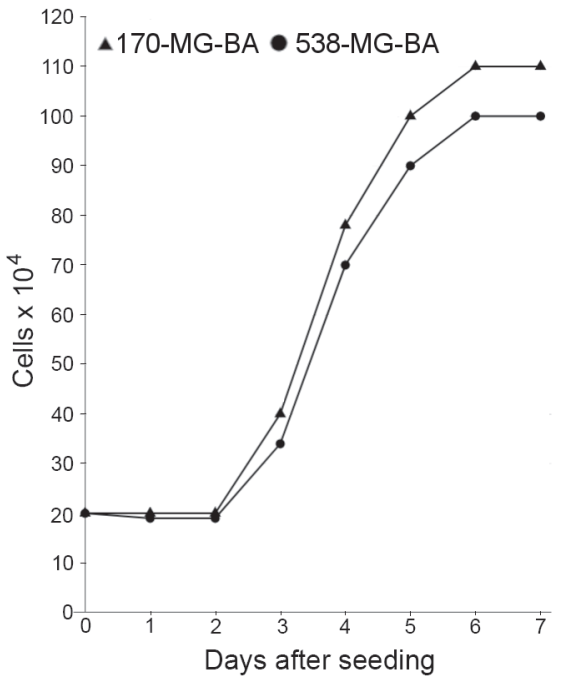

Figure 2: Growth curves of 170-MG-BA and 538-MG-BA cell lines. of CK-positive cells appeared at PN 30-50. The majority of cells in both cell lines expressed nestin throughout, but these were NF-negative. Double labeling of 170-MG-BA cultures in early passages showed co-expression of GFAP/Vim, GFAP/ Nes, but Nes/CK were not co-expressed (Figures 3A-I).

The vast majority of cells in 170-MG-BA cell line expressed EGFR. Indirect immunofluorescence showed varied intensities of EGFR staining with a remarkable subpopulation (5 to $10 \%$ ) of strongly positive stained cells which occurred at both early (PN 15) and late (PN 50) passages (Figures 4 A, B). The 538-MG-BA cells, however, remained EGFR-negative throughout culture (Figure 4C). Double labeling for GFAP/ EGFR showed EGFR negative staining of GFAP-positive cells (Figures 4D-F).

The predominant cell population in 170-MG-BA cell line at PN 50 was: EGFR+/Vim+/Nes+, GFAP-/CK-; while the corresponding profile of 538-MG-BA cells was EGFR-/ Vim+/Nes+/GFAP-/CK- (Figures 5 A-F). 

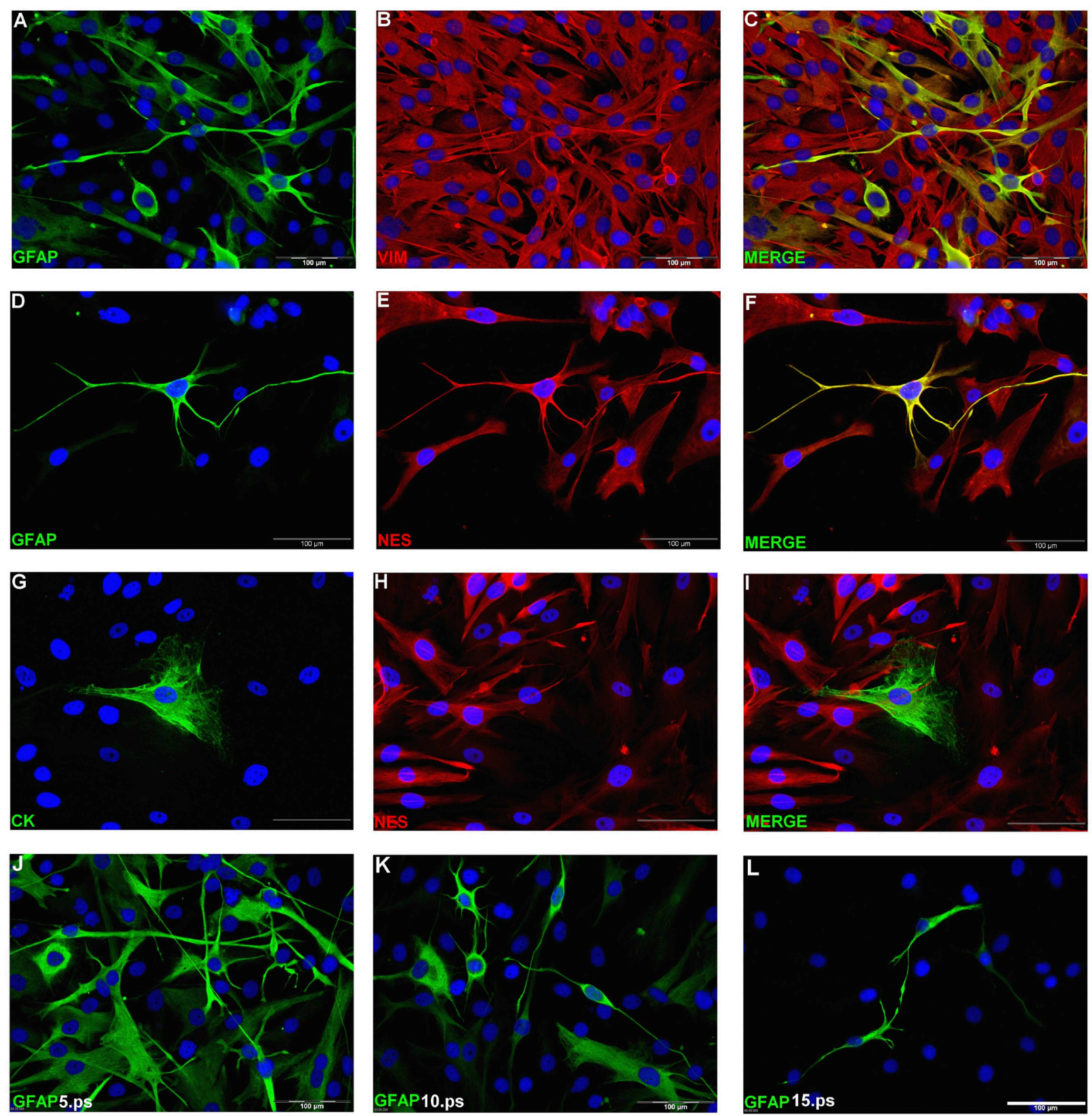

Figure 3. Double labeling (A-I) and indirect immunofluorescence $(\mathrm{J}-\mathrm{L})$ of 170-MG-BA in early passages. GFAP+/Vim+ (A-C), GFAP+/Nes+ (D-F), CK+/Nes- (G-I). Various morphologies of GFAP+ cells, their number decreased with increased PN (I-K). Nuclei stained with Hoechst. Scale bar: $100 \mu \mathrm{m}$.

Cell growth. Cell growth in tissue cultures prepared from 30 glioblastoma multiforme tissue explants exhibited high level of growth variability. Lethargic growth characterized 5 cultures and these had early proliferation arrest between PN 7-8. A further 23 cultures showed growth arrest by PN 10-15. Areas of rapidly proliferating cells appeared at PN $3-5$ in two of the 30 cultures. They gave rise to the permanent cell lines, designated 170-MG-BA and 538-MG-BA. Population doubling times were determined from growth curves (Figure 2), and were approximately 24 hours at PN
45-50 in 170-MG-BA and 28 hours in 538-MG-BA cell line. The numbers of cells in both cell lines remained static in the initial 48 hours, but neither cell line could tolerate higher cell dilution after passage. Hence, cultures with $1 \times 10^{4}$ cell density plated in $6 \mathrm{~cm}$ diameter dishes degenerated after several days.

Cytogenetics. Cytogenetic and key aCGH data for 170-MG-BA and 538-MG-BA are provided in Figures 6 and S1 . Both cell lines carried multiple chromosome rearrangements (Figure 6A, B), including homogeneously staining regions (hsr), indicated with red arrows, associated with focal gene 

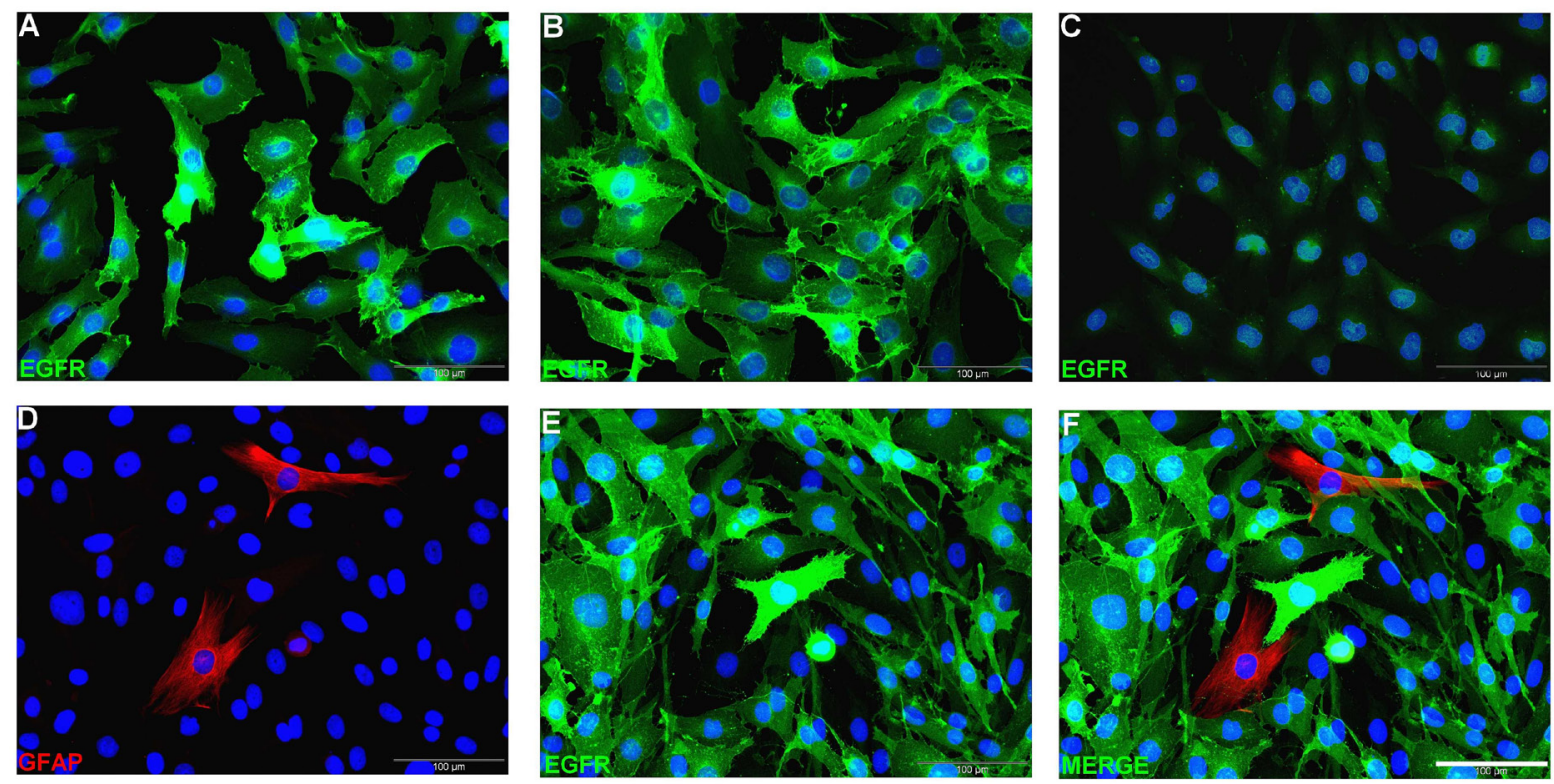

Figure 4. Indirect immunofluorescence for EGFR (A-C) and double labeling for GFAP/EGFR in glioblastoma cell lines (D-F). EGFR+ stained cells at PN 5 (A) and PN 50 (B) in 170-MG-BA and negative staining in 538-MG-BA (C) cell line. Double labeling for GFAP/EGFR showed EGFR negative staining of GFAP-positive cells (D-F) in 170-MG-BA cell line at PN 15. Nuclei stained with Hoechst. Scale bar: $100 \mu \mathrm{m}$.
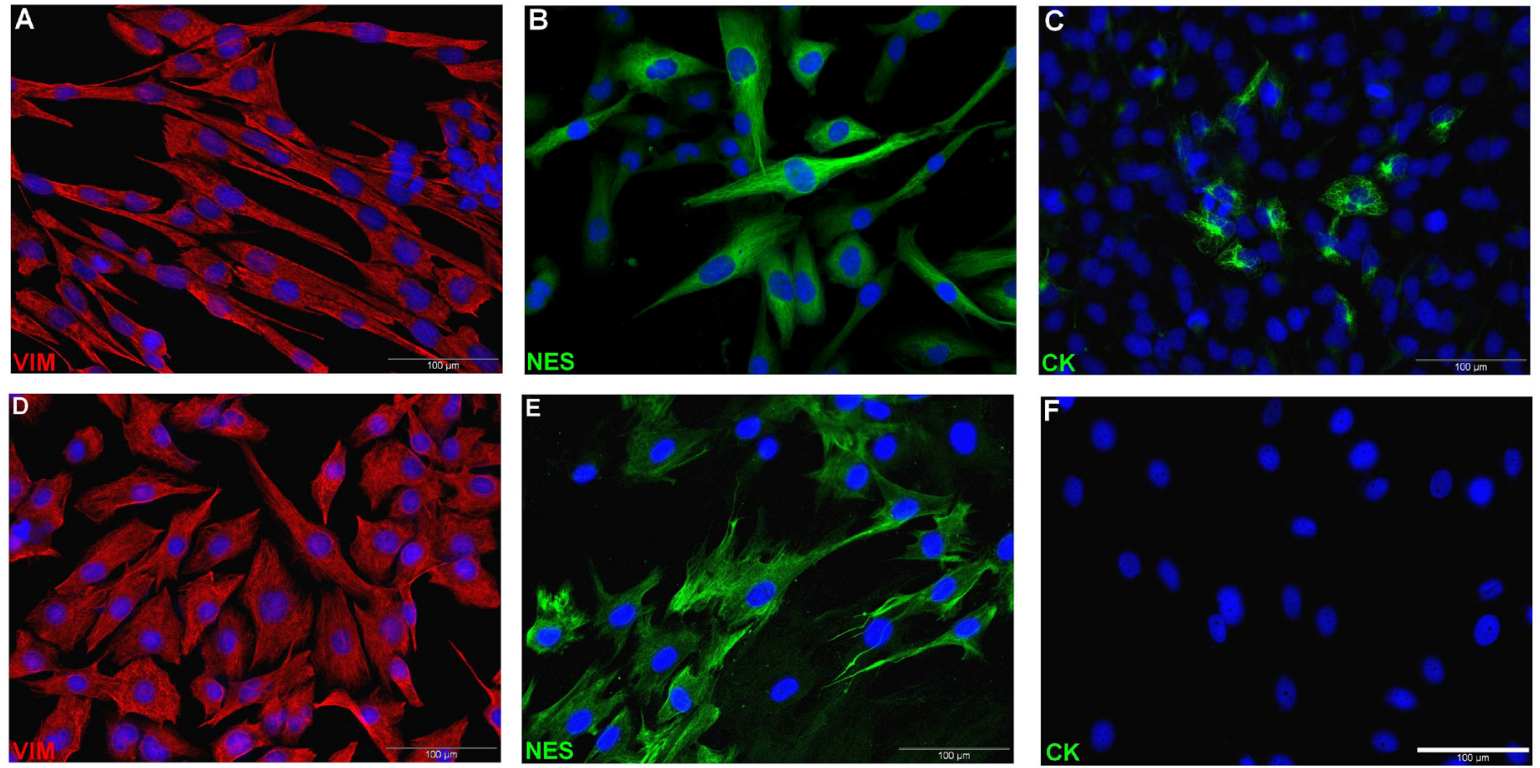

Figure 5. IF expression in cell lines at 45 to 50 passages, 538-MG-BA (A-C) and 170-MG-BA (D-F). Indirect immunofluorescence staining for vimentin (A/D), nestin (B/E) and CK (C/F). Nuclei stained with Hoechst. Scale bar: $100 \mu \mathrm{m}$.

amplification in paired $\operatorname{der}(17) t(7 ; 17)(7 ; 3)$ markers present in 170-MG-BA (Figure 6A). These markers were transmitted stably. FISH with a BAC clone covering the epidermal growth factor receptor gene EGFR (shown in magenta), together with whole chromosome paints for chromosomes 3 (gold) and 17 (green) confirmed EGFR amplification and revealed co-amplification with chromosome 3 (Figure S2c). aCGH delineated and quantified the amplicon, showing it to cover approximately $800 \mathrm{kbp}$ of chr 7 hosting EGFR (Figure 6d). Amplification of EGFR peaked at around 30-fold haploid copy number. No comparable amplicon was present on chromosome 3 (Figure S1a), obscuring the function of the co-amplified chromosome 3 material. aCGH also revealed multiple deleted regions and losses in heterozygosity (Figure S1). Biallelic focal deletions were present in both 170-MG-BA and 538-MG-BA affecting CDKN-2A/B (Figure 6E). Larger 

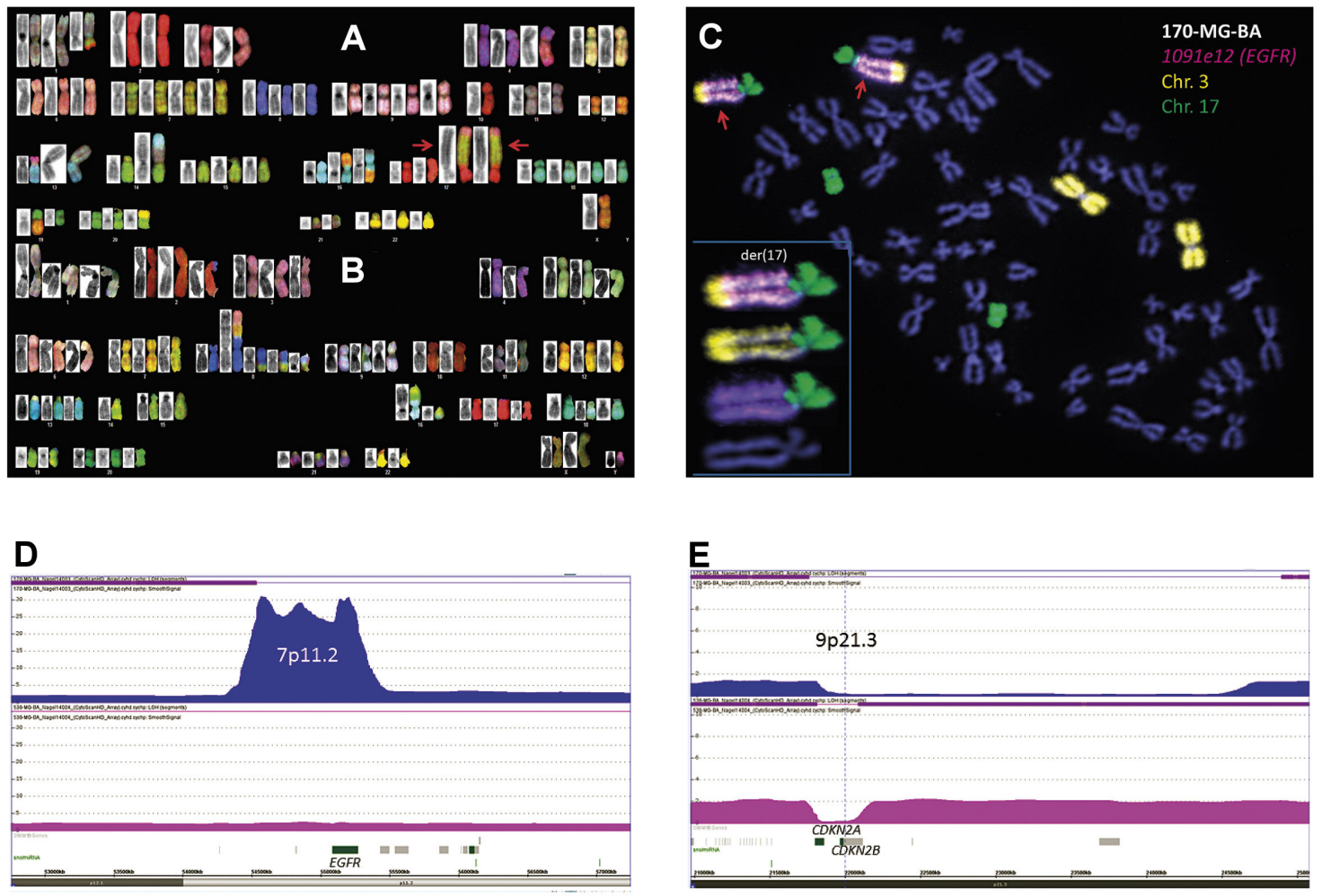

Figure 6. Genomic analysis. Shows SKY analysis of 170-MG-BA (A) and 538-MG-BA (B). Paired chromosome images show raw SKY (left) and reverse G-banding for each homologue. Note hsr deriving from chromosome 7 material (red arrows) marking a paired der(17) in 170-MG-BA. FISH probe colors were enhanced for clarity. C) FISH shows amplification of EGFR region (magenta). Inset shows co-amplification with chromosome 3 material (gold) on paired der(17) marker (green). D) aCGH confirms parsimonious targeting of focal amplicon at 7p11.2 ( 30×) bearing EGFR. E) aCGH reveals focal deletion of CDKN2-A/B in both 170-MG-BA (blue) and 538-MG-BA (red).

shared monoallelic deletions present on chromosome $15 \mathrm{q}$ of approximately $9.6 \mathrm{Mbp}(40.7-50.3 \mathrm{Mbp})$ and $22 \mathrm{q}$ of $3.8 \mathrm{Mbp}$ (39.6-43.4 Mbp) are shown in Figure S1.

Gene expression. RQ-PCR analysis confirmed that EGFR was uniquely highly expressed in 170-MG-BA cells with 7q11 amplification, dwarfing HeLa reference positive control cells $>40$-fold (Figure 7A). In contrast, the EGFR expression in 538-MG-BA cells which lacked amplification was not elevated. Targeted expression analysis revealed that neither 170-MG-BA nor 538-MG-BA showed signals for EGFRvIII. The TBP endogenous control was positive, indicating that both the cDNA and EGFRvIII signals for the vector control were intact, thus proving the EGFRVIII assay functional.

\section{Discussion}

Herein, we report the process of glioma cell line establishment from 30 histologically diagnosed glioblastoma multiforme IV tumor brain samples and characterization of the 170-MG-BA and 538-MG-BA originated cell lines - the former is the first conventional cell line with high-level EGFR amplification and expression.

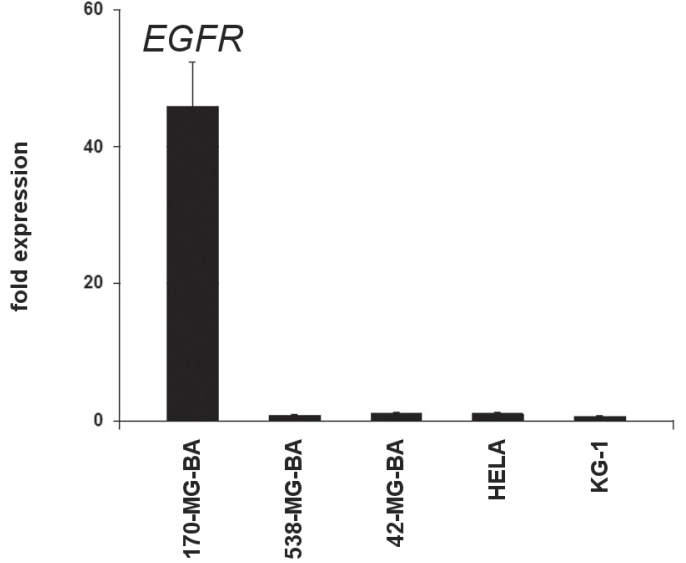

Figure 7. Gene expression. RqPCR shows massive (>40-fold) EFRR upregulation in 170-MG-BA compared to positive control HELA cells and 538-MG-BA cells.

While the remaining 28 tissue cultures showed limited life spans and underwent senescence between PN 7-15, 170-MG-BA and 538-MG-BA were successfully cultivated up to at least passage number 50 , consistent with establish- 
ment/immortalization. Both cell lines originated with similar dynamics when areas of nascent cultures bearing smaller, more rapidly proliferating cells emerged and overgrew the remaining flat and process-bearing cells. These findings support the notion that only a relatively small fraction of cells, sometimes equated with cancer stem cells, possess the ability to proliferate and self-renew. Both newly established cell lines showed similar growth rates, moderate morphological differences and cytoskeletal heterogeneity. We found wide differences in GFAP immunostaining between the established cell lines: morphologically variable GFAPpositive cells dominated 170-MG-BA cultures up to PN5. Their number slowly decreased and completely disappeared after PN 20. In contrast, GFAP-positive cells were absent from 538-MG-BA cultures.

GFAP expression is frequently lost with increasing grade of glioma malignancy. Most permanent human glioma cell lines immunostain negatively for GFAP or lose GFAP expression after serial passaging [22]. Although specific for normal and neoplastic epithelial cells, cytokeratins (CK) are found in glioma tissue [23]. Recently we described a subpopulation of CK-positive cells in previously established glioblastoma cells lines [24] and also in normal human "glial-like" cells [25].

Although a 3-5\% CK-positive subpopulation arose in the 538-MG-BA cell line at higher passages, 170-MG-BA CK-positive cells appeared only in early passages, where mainly flat cells were positively stained. The unexpected presence of CK in glioma tissue, glioma cell lines and cultured human "glial-like" cells is most likely connected with glial cell ectodermal origin. Nestin is an IF protein originally identified as a marker for neuro-epithelial precursor cells and nestin is currently considered a marker for neural stem cells [26]. We detected a high percentage of nestin positive cells in both cell lines, and IF protein assessment confirmed intra and inter-heterogeneity of glioblastoma cell lines. The combination of these and our previous studies $[25,27]$ suggest it is instructive to reconsider the cell-type specificity of IF under culture conditions.

STR profiling confirmed the uniqueness of both cell lines and proved their authenticity. This was bolstered by congruent patient and cell line EGFR activation in 170-MG-BA cells. Combined cytogenetic, genomic and transcriptional analyses identified stable high level EGFR expression in 170-MG-BA cells; apparently the first such conventional glioma cell line [9]. Immunostaining confirmed protein expression of EGFR in 170-MG-BA only. Since EGFR is upregulated in approximately $50 \%$ of glioblastoma patients, 170-MG-BA is a uniquely fit pre-clinical model for investigation of novel targeted therapies; especially because both the effectiveness of small molecule EGFR inhibitors [28,29] and the search for biomarkers predictive of therapeutic responses have so far proved disappointing [30]. EGFR ${ }^{\mathrm{HIGH}}$ stability of 170-MG-BA may reflect genomic amplification via chromosomally integrated hsr instead of (unstable) extra-chromosomal double minute chromosomes which can be lost during mitosis, or the presence of alterations affecting other genes, such as CDKN2A/B. 170-MG-BA tested negative for EGFRvIII, and since EGFRvIII is the most frequent, but not the only mutation variant co-expressed in EGFR ${ }^{\mathrm{HIGH}}$ glioblastoma cells, expression of another rarer variant cannot be excluded [31].

DNA STR profiles were both unique when compared with the global database available for interactive scrutiny at the DSMZ and ATCC, JCRB, and RIKEN repositories. Recent retrospective studies have cast doubt on the identity and stability of several classic glioma cell lines $[14,15,16]$, while others, such as SKMG-3, are subject to restricted distribution. The lack of a suitable and practical in vitro model has hampered investigation into the biology of EGFR ${ }^{\mathrm{HIGH}}$ which is the most frequent genomic alteration in malignant glioma and a major contributor to treatment resistance [1]. EGFR amplification is apparently unstable in conventional cell lines, with only the SKMG-3 glioma cell line described and only at moderate amplification levels [7]. This has hampered key investigations, such as identifying pathways disturbed by $\mathrm{EGFR}^{\mathrm{HIGH}}$ and how to mitigate them. Hence, provision of the newly authenticated $170-\mathrm{MG}-\mathrm{BA} \mathrm{EGFR}^{\mathrm{HIGH}}$ glioma cell line fills the yawning gap in clinical armamenta.

Finally, cell lines 170-MG-BA (ACC 804) and 538-MG-BA (ACC 805) have been deposited at the DSMZ cell bank, thus providing novel authenticated pre-clinical tools freely available to qualified investigators.

Supplementary information is available in the online version of the paper.

Acknowledgements: We thank Mrs. Kaufmann, Hillova, Skopekova, Galfyova and Mr. Miklos for expert technical assistance.

\section{References}

[1] HATANPAA KJ, BURMA S, ZHAO D, HABIB AA. Epidermal growth factor receptor in glioma: signal transduction, neuropathology, imaging, and radioresistance. Neoplasia 2010; 12: 675-684.

[2] PADFIELF E, ELLIS HP, KURIAN KM. Current therapeutic advances targeting EGFR and EGFRvIII in glioblastoma. Front Oncol 2015; 5: 5. https://doi.org/10.3389/ fonc.2015.00005

[3] WESTPHAL M, MAIRE CL, LAMSYUS K. EGFR as a target for glioblastoma treat-ment: An unfulfilled promise. CND Drugs 2017; 31: 723-735. https://doi.org/10.1007/s40263017-0456-6

[4] LIFFERS K, LAMSZUS K, SCHULTE A. EGFR amplification and glioblastoma stem-like cells. Stem Cells Int 2015; 2015: 427518. https://doi.org/10.1155/2015/427518

[5] Del VECCHIO CA, GIACOMINI CP, VOGEL H, JENSEN KC, FLORIO T et al. EGFRvIII gene rearrangement is an early event in glioblastoma tumorigenesis and expression defines a hierarchy modulated by epigenetic mechanisms. Oncogene 2013; 32: 2670-2681. https://doi.org/10.1038/onc.2012.280 
[6] STEC WJ, ROSIAK K, SIEJKA P, PECIAK J, POPEDA M et al. Cell line with endogenous EGFRvIII expression is a suitable model for research and drug development purposes. Oncotarget 2016; 7: 31907-31925. https://doi.org/10.18632/ oncotarget.8201

[7] SCHULTE A, LIFFERS K, KATHAGEN A, RIETHDORF S, ZAPF $S$ et al. Erlotinib resistance in EGFR-amplified glioblastoma cells is associated with upregulation of EGFRvIII and PI3Kp110d. Neuro Oncol 2013; 15: 1289-1301. https:// doi.org/10.1093/neuonc/not093

[8] PANDITA A, ALDAPE KD, ZADEH G, GUHA A, JAMES $\mathrm{CD}$. Contrasting in vivo and in vitro fates of glioblastoma cell subpopulations with amplified EGFR. Genes Chromosomes Cancer 2004; 39: 29-36. https://doi.org/10.1002/gcc.10300

[9] SCHULTE A, GUNTHER HS, MARTENS T, ZAPF S, RIETHDORF S et al. Glioblastoma stem-like cell lines with either maintenance or loss of high-level EGFR am-plification, generated via modulation of ligand concentration. Clin Cancer Res 2012; 18: 1901-1913. https://doi.org/10.1158/10780432.CCR-11-3084

[10] FAN QW, CHENG CK, GUSTAFSON WC, CHARRON E, ZIPPER P et al. EGFR phosphorylates tumor-derived EGFRvIII driving STAT3/5 and progression in glioblastoma. Cancer Cell 2013; 24: 438-449. https://doi.org/10.1016/j. ccr.2013.09.004

[11] GUO G, GONG K, WOHLFELD B, HATANPAA KJ, ZHAO $\mathrm{D}$ et al. Ligand-Independent EGFR Signaling. Cancer Res 2015; 75: 3436-3441. https://doi.org/10.1158/0008-5472. CAN-15-0989

[12] ZADEH G, BHAT KP, ALDAPE K. EGFR and EGFRvIII in glioblastoma: partners in crime. Cancer Cell 2013; 24: 403404. https://doi.org/10.1016/j.ccr.2013.09.017

[13] MACLEOD RAF, DIRKS WG, DREXLER HG. Where have all the cell lines gone? Int J Cancer 2013; 132: 1232-1234. https://doi.org/10.1002/ijc.27752

[14] VENTER DJ, STRATTON MR, NOBLE MD. Molecular genetic study showing that the IN/157 'oligodendroglioma' cell line has been contaminated by rhabdomyosarcoma (RD) cells. Neuropathol Appl Neurobiol 1992; 18: 159-169.

[15] CAPES-DAVIS A, THEODOSOPOULOS G, ATKIN I, DREXLER HG, KOHARA A et al. Check your cultures! A list of cross-contaminated or misidentified cell lines. Int J Cancer 2010; 127: 1-8.

[16] BADY P, DISERENS AC, CASTELLA V, KALT S, HEINIMANN $\mathrm{K}$ et al. DNA fingerprinting of glioma cell lines and considerations on similarity measurements. Neuro Oncol 2012; 14: 701-711. https://doi.org/10.1093/neuonc/nos072

[17] MACLEOD RAF, DIRKS WG, MATSUO Y, KAUFMAN M, MILCH H et al. Wides-pread intraspecies cross-contamination of human tumor cell lines arising at source. Int J Cancer 1999; 83: 555-563.

[18] MACLEOD RAF, KAUFMANN ME, DREXLER HG. Harvesting of Cancer Cells and Cell Lines. Methods Mol Biol 2017; 1541: 43-58. https://doi.org/10.1007/978-1-4939-6703-2_5

[19] CHEN S, NAGEL S, SCHNEIDER B, DAI H, GEFFERS R et al. A new ETV6-NTRK3 cell line model reveals MALAT1 as a novel therapeutic target - a short re-port. Cell Oncol (Dordr). 2018;41:93-101.https://doi.org/10.1007/s13402-017-0356-2
[20] DAI HP, EHRENTRAUT S, NAGEL S, EBERTH S, POMMERENKE C et al. Ge-nomic Landscape of Primary Mediastinal B-Cell Lymphoma Cell Lines. PLoS One 2015; 10: e0139663. https://doi.org/10.1371/journal.pone.0139663

[21] DIRKS WG, DREXLER HG. STR DNA typing of human cell lines: detection of intra- and interspecies cross-contamination. Methods Mol Biol 2013; 946: 27-38. https://doi. org/10.1007/978-1-62703-128-8_3

[22] RESTREPO A, SMITH CA, AGNIHOTRI S, SHEKARFOROUSCH M, KONGKHAM PN et al. Epigenetic regulation of glial fibrillary acidic protein by DNA methylation in human malignant gliomas. Neuro Oncol 2011; 13: 42-50. https://doi.org/10.1093/neuonc/noq145

[23] HIRATO J, NAKAZATO Y, OGAWA A. Expression of nonglial intermediate fila-ment proteins in gliomas. Clin Neuropathol 1994; 13: 1-11.

[24] MACIKOVA I, PERZELOVA A, MRAZ P, STENO J, BIZIK I. Heterogeneity of keratin intermediate filaments expression in human glioma cell lines. Neoplasma 1999; 46: 390393.

[25] SIVAKOVA I, PERZELOVA A, KUBIKOVA E, MRAZ P. Unexpected presence of cytokeratins in human "glia-like"cells. Bratisl Med J 2013; 114: 274-278.

[26] ZHANG M, SONG T, YANGL, CHEN R, WU L et al. Nestin and CD133: valuable stem cell-specific markers for determining clinical outcome of glioma patients. J Exp Clin Cancer Res 2008; 27: 85. https://doi.org/10.1186/1756-9966-2785

[27] PERZELOVA A, MACIKOVA I, TARDY M, MRAZ P, BIZIK I et al. Subpopulation of nestin positive glial precursor cells occur in primary adult human brain cultures. Biologia 2007; 62: 633-640.

[28] PEEREBOOM DM, SHEPARD DR, AHLUWALIA MS, BREWER CJ, AGARWAL N et al. Phase II trial of erlotinib with temozolomide and radiation in patients with newly diagnosed glioblastoma multiforme. J Neurooncol 2010; 98: 93-99. https://doi.org/10.1007/s11060-009-0067-2

[29] AHLUWALIA MS, XIE H, DAHIYA S, HASHEMI-SADRAEI N, SCHIFF D et al. Efficacy and patient-reported outcomes with dose-intense temozolomide in patients with newly diagnosed pure and mixed anaplastic oligodendroglioma: a phase II multicenter study. J Neurooncol 2015; 122: 111-119. https://doi.org/10.1007/s11060-014-1684-y

[30] MELLINGHOFF IK, WANG MY, VIVANCO I, HAASKOGAN DA, ZHU S et al. Molecular determinants of the response of glioblastomas to EGFR kinase inhibitors. N Engl J Med 2005; 353: 2012-2024. https://doi.org/10.1056/NEJMoa051918

[31] KASTENBURGER ER, HUSE JT, BERMAN SH, PEDRAZA A, ZHANG Y et al. Quantitative assessment of intragenic receptor tyrosine kinase deletions in primary glioblastomas: their prevalence and molecular correlates. Acta Neuropathol 2014; 127: 747-759. https://doi.org/10.1007/s00401-013$1217-3$ 


\section{High level EGFR amplification in a newly established glioblastoma cell line 170-MG-BA}

R. A. F. MACLEOD ${ }^{1}$, B. SCHNEIDER ${ }^{2}$, I. SIVAKOVA 3 , S. NAGEL ${ }^{1}$, W. G. DIRKS ${ }^{1}$, P. MRAZ ${ }^{3}$, E. KUBIKOVA ${ }^{3}$, A. PERZELOVA ${ }^{3, *}$

\section{Supplemental Material}

Supplementary Table 1. Primer sequences for mutation detection.

\begin{tabular}{llll}
\hline & Forward & Reverse & Probe \\
\hline EGFRvIII & gagtcgggctctggaggaa & ccacaggctcggacgcac & 6FAM - agccgtgatctgtcaccacataattacctttc - TMR \\
TBP & tcggagagttctgggattgt & cacgaagtgcaatggtcttt & 6FAM - accgcagctgcaaaatattgtatccacagt - TMR \\
\hline
\end{tabular}
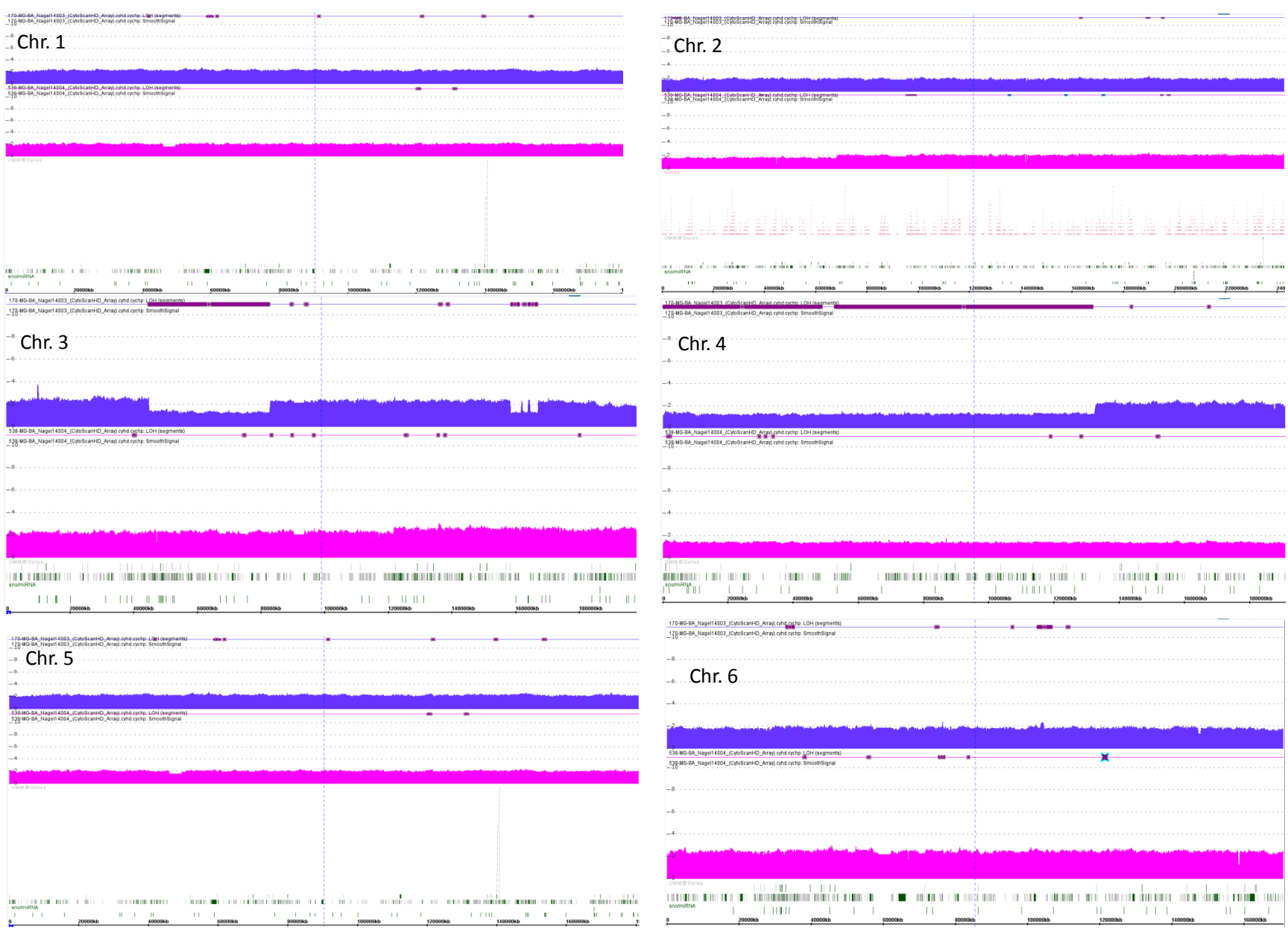

Supplementary Figure 1. Genomic array data. Shows copy number plots for chromosomes 1-22, X and Y for 170-MG-BA (blue) and 538-MG-BA (red). LOH plots are shown above in dark red. 


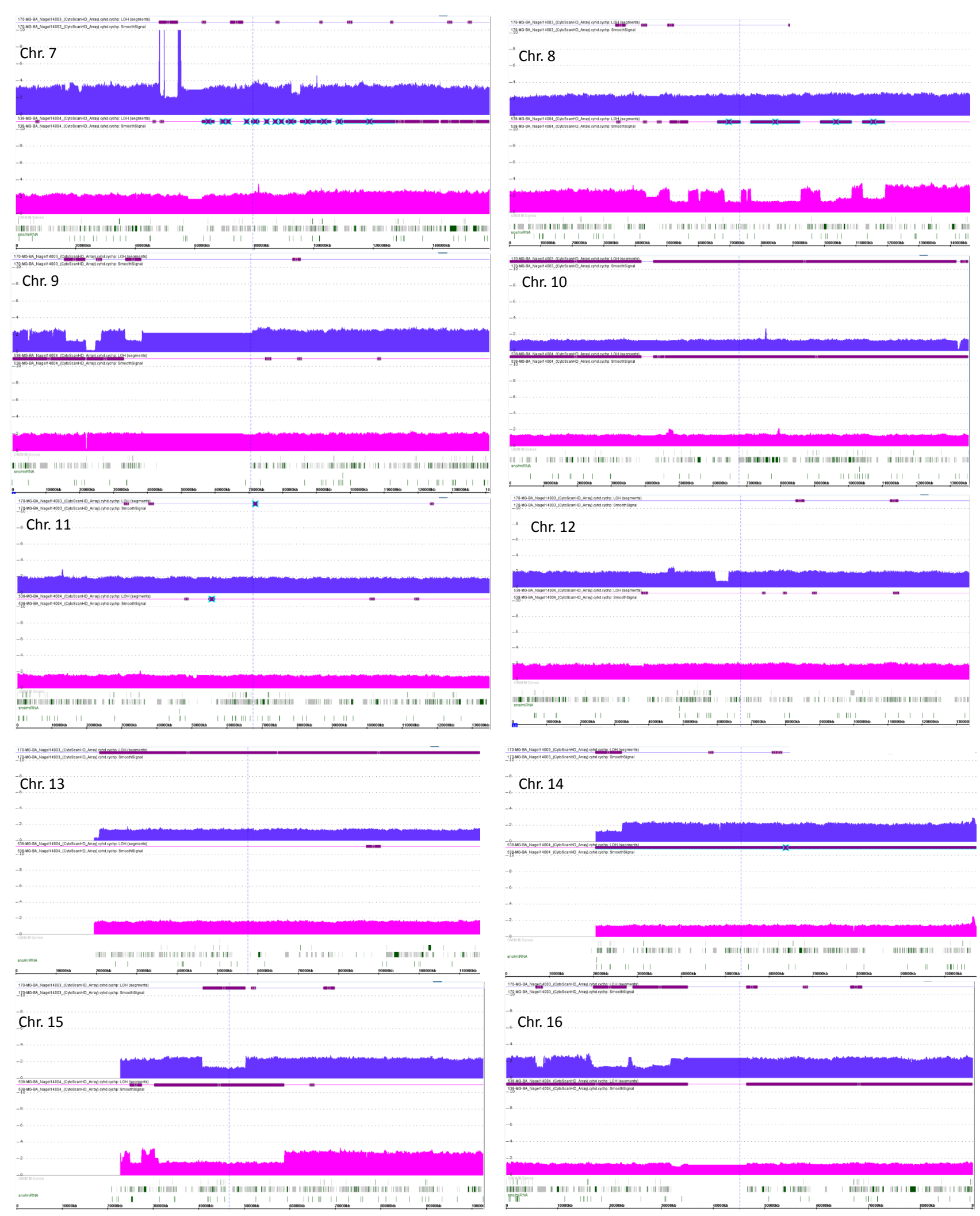

Supplementary Figure 1 cont. Genomic array data. Shows copy number plots for chromosomes 1-22, X and Y for 170-MG-BA (blue) and 538-MG-BA (red). LOH plots are shown above in dark red. 


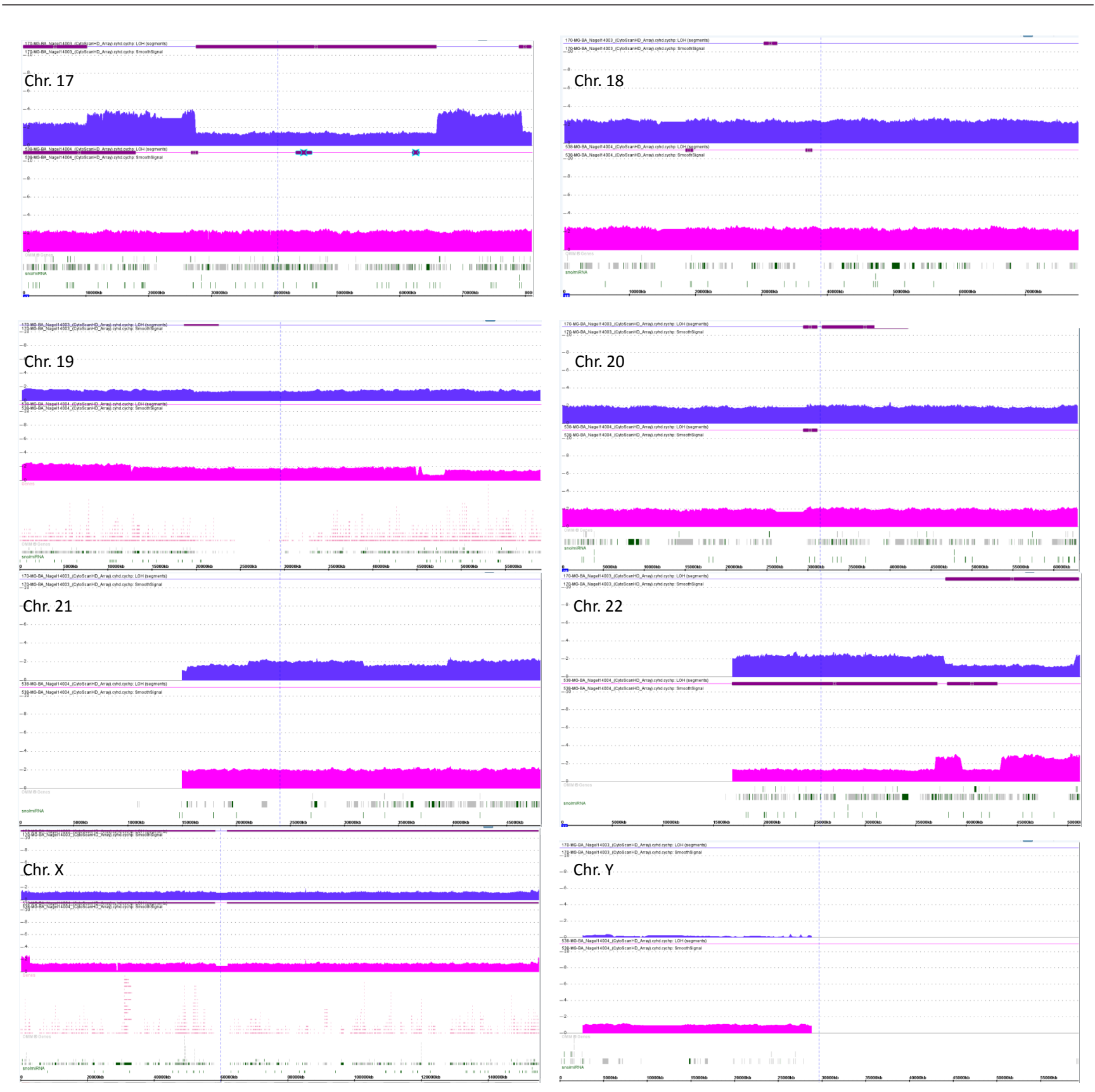

Supplementary Figure 1 cont. Genomic array data. Shows copy number plots for chromosomes 1-22, X and Y for 170-MG-BA (blue) and 538-MG-BA (red). LOH plots are shown above in dark red. 